\title{
Analyse vibro-acoustique des structures sandwich en NIDA micro-perforées utilisées dans le spatial
}

\author{
Abderrazak Omrani ${ }^{1, a}$, Raoul De Moura ${ }^{1}$ et Olivier Tanneau ${ }^{2}$ \\ 1 Laboratoire d'ingénierie des systèmes mécaniques et des matériaux (LISMMA), SUPMECA Paris, \\ 3 rue Fernand Hainaut, 93407 Saint-Ouen Cedex, France \\ 2 ALTRAN AIT, 2 rue Paul Vaillant Couturier, 92300 Levallois-Perret, France
}

Reçu le 30 septembre 2008, accepté le 18 décembre 2008

\begin{abstract}
Résumé - Les antennes paraboliques ultra légères des satellites de télécommunication, sont fabriquées en matériaux composites à partir de coques multicouches très minces obtenues par tissage. Pendant la première phase de vol du lanceur, lors de la traversée des premières couches atmosphériques, les excitations vibro-acoustiques dues au système de propulsion et aux forces aérodynamiques sont les plus critiques. La charge acoustique de type aléatoire induite sur la structure de l'antenne, devient alors très importante et peut provoquer des dommages aux structures et aux équipements. Pour réduire les charges acoustiques, les concepteurs utilisent des coques micro-perforées pour permettre à la structure de « respirer » et de réduire ainsi la charge acoustique. Le dimensionnement et l'optimisation de ces structures nécessitent des outils de calcul numérique. La prise en compte de la perforation du matériau pour le calcul de la charge induite par l'excitation acoustique aléatoire n'est pas classique. L'objet de cette étude est de proposer un modèle d'impédance locale représentatif de la micro-perforation pouvant être utilisé dans un code de calcul vibro-acoustique.
\end{abstract}

Mots clés : Structures micro-perforées / vibro-acoustique / calcul numérique

\begin{abstract}
Vibro-acoustic analysis of micro-perforated composite structures used in space craft industry. In tele-communication satellite, the light weight parabolic reflectors are made with multi layered composite materials. During the lift off of the launcher and the first stage of the flight, the vibroacoustic excitations due to the thrust system and to the aerodynamic forces are the most critical. The random acoustic load applied on the antenna structure becomes very important and can cause damage to the structure and their equipments. To reduce the acoustic loads, micro perforated thin shell structures are used in order to reduce the acoustic load applied to the structure. CAD and numerical software tools are needed to design and optimise such structure. The computation of the acoustic load induced by a diffuse field on a micro perforated structure is not classical. The aim of this study is to develop a numerical local impedance model to represent the effect of the perforation to be used within vibro-acoustic software.
\end{abstract}

Key words: Micro perforated structure / vibro-acoustics / numerical modelling

\section{Introduction}

Dans l'industrie spatiale, les structures sont souvent fabriquées avec des matériaux composites très minces et très légers. En particulier dans les satellites de télécommunication, les antennes paraboliques ultra légères sont fabriquées en matériaux composites sandwich avec un cœur NIDA recouvert de coques multicouches très minces et perforées obtenus par tissage.

a Auteur pour correspondance :

abderrazak.omrani@supmeca.fr
Ce procédé de fabrication permet d'une part, une réduction importante de la masse de la structure et d'autre part, une réduction considérable de la charge acoustique appliquée à la structure de l'antenne. En effet, pendant la première phase de vol du lanceur, lors de la traversée des premières couches atmosphériques, les excitations vibro-acoustiques dues au système de propulsion et aux forces aérodynamiques sont les plus critiques et peuvent atteindre et dépasser $140 \mathrm{~dB}$. La charge acoustique de type aléatoire induite sur la structure de l'antenne, communément connue sous le nom de « pression paroi bloquée », devient alors très importante, ce 
qui peut provoquer des dommages aux structures et aux équipements, d'où l'intérêt des perforations qui permettent à la structure de « respirer » et diminuer ainsi la charge acoustique appliquée à la structure. L'usage de cette technologie pour les antennes ultra légères est limité aux bandes de fréquences $\mathrm{Ku}(12-14 \mathrm{GHz})$ à cause des dimensions des perforations comprises entre 1 et $2 \mathrm{~mm}$. Pour couvrir des fréquences d'émission plus élevées dans la bande $\mathrm{Ka}$ (20-30 GHz), il est nécessaire de réduire la taille des perforations, ce qui aura comme effet d'augmenter sensiblement la charge acoustique à travers l'antenne.

Il est donc important de disposer d'outils de calcul et d'aide à la conception pour permettre de faire de la prédiction dans le domaine vibro-acoustique.

La modélisation des structures en matériaux composites est basée sur la technique d'homogénéisation qui est bien établie et les codes de calcul de structures sont nombreux (Nastran, Ansys, Abaqus, ...). Par contre en acoustique, la prise en compte de la perforation du matériau pour le calcul de la charge induite par l'excitation acoustique aléatoire n'est pas classique.

Après un bref rappel des équations de base du problème physique et des principales hypothèses ayant permis d'établir le modèle d'impédance des plaques micro-perforées (PMP) [3], le but de ce travail est de proposer un modèle analytique d'impédance locale adapté à une structure composite en NIDA micro-perforée à partir du modèle pour les plaques simples micro-perforées disponible dans la littérature. Ce modèle analytique sera validé expérimentalement par des essais en tube de Kundt.

\section{Application des PMP pour l'isolation acoustique}

L'utilisation des plaques micro-perforées pour l'isolation acoustique constitue une alternative très intéressante à l'utilisation des matériaux poreux classiques (mousses, laines de verre, laine de roche, etc.), à chaque fois que ces derniers matériaux ne peuvent être utilisés pour des raisons de sécurité et/ou pour des raisons fonctionnelles. En effet les matériaux poreux classiques sont des matériaux opaques et généralement inflammables.

Les domaines d'application des PMP sont multiples allant du bâtiment et de l'urbanisme jusqu'aux industries aéronautiques et spatiales, en passant par les industries automobiles et du transport. Par exemple, l'usage de panneaux de PMP translucides peut améliorer le confort acoustique d'un bâtiment sans pour autant empêcher la lumière naturelle. Les PMP sont aussi utilisées avantageusement pour la réduction du bruit des silencieux des véhicules et des nacelles de moteurs d'avions fonctionnant à hautes températures.

Le principe de fonctionnement des PMP est simple. Lorsqu'une structure micro-perforée est excitée par une onde acoustique incidente, celle-ci va se propager dans les perforations et va perdre ainsi une partie de son énergie qui est dissipée par frictions et échange de chaleur.

\subsection{Les différents effets intervenant dans la dissipation}

Les perforations des plaques peuvent être assimilées à des tubes rigides de petits diamètres. Les effets thermovisqueux sur les parois ne peuvent donc pas être négligés $\mathrm{du}$ fait des dimensions relativement voisines des tubes avec celles des couches limites visqueuses et thermiques.

\section{- Effet de la viscosité de l'air}

Pour des diamètres de perforations inférieurs au mm, l'air ne peut plus être considéré comme un fluide parfait. La viscosité de l'air agit à proximité des parois où un fort gradient de vitesse se développe dans une épaisseur appelée couche limite visqueuse. Son épaisseur dépend de la fréquence et des propriétés de viscosité du fluide de propagation : $d_{\mathrm{visc}}=\sqrt{\frac{2 \nu}{\omega}}$, où $\nu=\frac{\mu}{\rho_{0}}$ est la viscosité cinématique, $\omega$ est la pulsation, $\mu$ et $\rho_{0}$ sont respectivement la viscosité dynamique et la masse volumique du fluide. Pour l'air, dans les conditions normales, ces épaisseurs sont comprises entre 0,5 et $0,015 \mathrm{~mm}$ pour les fréquences comprises entre $20 \mathrm{~Hz}$ et $20 \mathrm{kHz}$.

La dissipation concentrée dans cette couche limite est la principale source de dissipation des systèmes de plaques micro-perforées mais aussi de la plupart des matériaux absorbants poreux.

\section{- Effet de la conduction thermique}

L'effet de la conduction thermique est caractérisé par la vitesse laminaire entropique du fluide proche des parois. Cet effet est non négligeable uniquement dans la couche limite thermique. L'épaisseur de cette couche limite est donnée par : $d_{\text {therm }}=\sqrt{\frac{\nu^{\prime}}{\omega}}$, où $\nu^{\prime}=\frac{\kappa}{\rho_{0} C_{\nu}}, \kappa$ est le coefficient de conductivité thermique et $C_{\nu}$ la capacité calorifique à volume constant. Pour l'air dans les conditions normales, ces épaisseurs sont comprises entre 0,58 et $0,02 \mathrm{~mm}$ pour les fréquences comprises entre $20 \mathrm{~Hz}$ et $20 \mathrm{kHz}$.

Ces effets thermiques sont le plus souvent négligés pour les plaques perforées de faibles épaisseurs.

\section{Modèle mathématique de l'acoustique des PMP}

Plusieurs approches théoriques, empiriques et expérimentales ont été utilisées pour la modélisation de l'absorption acoustique des plaques micro-perforées (PMP). Les premières études remontent à Crandall [2], Sivian [7], Ingard [4], Thurston [10] et Melling [6].

Des travaux plus récents ont repris ces études et proposé de nouveaux modèles. À titre d'exemple, on peut citer les travaux de Stinson [8,9] qui a proposé des approximations plus simples trouvées numériquement à partir des équations de Kirchhoff jugées plus difficiles à utiliser. Plus récemment encore, Thomas Dupond [3] dans sa 
thèse, s'est intéressé non seulement à l'absorption acoustique des plaques micro-perforées mais aussi à la transparence de celles-ci. Dans cette thèse on peut trouver une bonne synthèse bibliographique sur les travaux antérieurs réalisés dans le domaine.

\section{1 Équations de Kirchhoff}

Les équations proposées par Kirchhoff décrivant au premier ordre la thermomécanique d'un gaz soumis à de petites perturbations sont les suivantes $[3,9]$ :

$$
\begin{aligned}
\rho_{0} \frac{\partial \vec{\nu}}{\partial t} & =-\vec{\nabla} P_{\mathrm{a}}+\frac{4}{3} \mu \vec{\nabla}(\vec{\nabla} \bullet \vec{\nu})-\mu \vec{\nabla} \times \vec{\nabla} \times \vec{\nu} \\
\frac{\partial \rho}{\partial t} & =-\rho_{0} \vec{\nabla} \bullet \vec{\nu} \\
\kappa \Delta T & =\frac{T_{0}}{P_{0}}\left(\rho_{0} C_{\nu} \frac{\partial P_{\mathrm{a}}}{\partial t}-P_{0} C_{\mathrm{p}} \frac{\partial \rho}{\partial t}\right) \\
\frac{\partial P_{\mathrm{a}}}{\partial t} & =\frac{P_{0}}{\rho_{0} T_{0}}\left(\rho_{0} \frac{\partial T}{\partial t}+T_{0} \frac{\partial \rho}{\partial t}\right)
\end{aligned}
$$

Les équations (1)-(4) traduisent respectivement, la conservation de la quantité de mouvement et de la masse, la conductivité thermique d'un gaz et la loi d'état. $P_{\mathrm{a}}$, $\vec{\nu}, \rho, T$ sont la pression absolue, la vitesse acoustique, la masse volumique du gaz et sa température. $P_{0}, \rho_{0}$ et $T_{0}$ sont les grandeurs à l'équilibre, $\mu$ et $\kappa$ sont la viscosité dynamique et la conductivité thermique du fluide, $C_{\nu}$ et $C_{\mathrm{p}}$ sont les capacités calorifiques à pression et volume constant. La viscosité cinématique est donnée par $\nu=\frac{\mu}{\rho_{0}}$, en introduisant $\nu^{\prime}=\frac{\kappa}{\rho_{0} C_{\nu}}$ une constante de transfert thermique et $\gamma=\frac{C_{\mathrm{p}}}{C_{\nu}}$ la constante d'un gaz parfait, les relations suivantes sont données :

$$
\begin{cases}C_{\mathrm{p}}-C_{\nu} & =\frac{P_{0}}{\left(\rho_{0} T_{0}\right)} \\ \rho_{0} c^{2} & =\gamma P_{0}\end{cases}
$$

où $c$ désigne la célérité du son dans le fluide.

Pour l'application au cas d'un tube cylindrique de rayon $R$ (diamètre $a=2 R$ ), les hypothèses suivantes sont ajoutées :

- l'excitation est supposée harmonique (convention $\left.\mathrm{e}^{j \omega t}\right)$;

- pression, température et densité évoluent avec de petites variations (respectivement $p, \tau$ et $\delta$ ) autour des équilibres $P_{0}, T_{0}$ et $\rho_{0}$, il n'y a pas d'écoulement continu $V_{0}=0$ ( $\nu$ est directement la vitesse acoustique);

- les conditions sur les bords du tube sont vitesse et excès de température nuls ;

- les équations du problème sont développées en coordonnées cylindriques et la composante tangentielle est supposée nulle.

Dans ces conditions la vitesse s'écrit sous la forme : $\vec{\nu}=$ $q \vec{r}+u \vec{z}$.
En plus des hypothèses précédentes, le modèle classique utilisé suppose les approximations suivantes sur la solution :

- composante radiale de la vitesse négligée par rapport à la composante axiale;

- variation de la vitesse axiale plus importante suivant l'axe radial que suivant l'axe $z$;

- pression constante sur la section de tube;

- découplage des effets thermiques et visqueux (Éqs. (1) et $(3))$.

Ces hypothèses ont été vérifées et justifiées théoriquement par Stinson [9] pour des tubes de section vérifiant $R f^{3 / 2}<$ $10^{6} \mathrm{~cm} . \mathrm{s}^{-3 / 2}$ et $R>10^{-3} \mathrm{~cm}$.

L'équation de conservation de la quantité de mouvement peut alors se réduire à l'expression [6] :

$$
j \omega \rho_{0} u-\frac{\mu}{r} \frac{\partial}{\partial r}\left(r \frac{\partial u}{\partial r}\right)=\frac{\partial P}{\partial z}
$$

Il s'agit d'une équation de Bessel dont la solution générale avec la condition limite de vitesse nulle en $r=R$ s'écrit :

$$
u(r)=\frac{\partial p}{\partial z} \frac{1}{j \omega \rho_{0}}\left(1-\frac{J_{0}\left(r \sqrt{\frac{-j \omega \rho_{0}}{\mu}}\right)}{J_{0}\left(R \sqrt{\frac{-j \omega \rho_{0}}{\mu}}\right)}\right)
$$

$J_{0}$ est la fonction de Bessel de première espèce d'ordre 0 .

Comme la pression et son gradient ne dépendent pas de $r$, une homogénéisation est effectuée par la moyenne du profil de vitesse :

$$
\langle u\rangle=\frac{1}{A} \oiint u(r) \mathrm{d} S=\frac{1}{\pi R^{2}} \int_{0}^{R} u(r) 2 \pi r \mathrm{~d} r
$$

Il vient alors une équation homogénéisée de type Euler :

$$
j \omega \rho_{0}\langle u\rangle=\frac{\partial p}{\partial z}\left(1-\frac{2}{x \sqrt{-j}} \frac{J_{1}(x \sqrt{-j})}{J_{0}(x \sqrt{-j})}\right)
$$

avec :

$$
x=\sqrt{\frac{\omega \rho_{0}}{\mu}} \frac{a}{2} .
$$

\subsection{Impédance acoustique d'une perforation}

En supposant le tube suffisamment court pour pouvoir simplifier le gradient de pression en : $\frac{\partial P}{\partial z}=\frac{\Delta P}{e},(e$ désigne l'épaisseur de la plaque), l'impédance acoustique d'une perforation se ramène à :

$$
Z_{\mathrm{p}}=j \omega \rho_{0} e\left(1-\frac{2}{x \sqrt{-j}} \frac{J_{1}(x \sqrt{-j})}{J_{0}(x \sqrt{-j})}\right)^{-1}
$$

De nombreux auteurs ont proposé des simplifications supplémentaires permettant de remplacer les fonctions de Bessel par des expressions plus simples. Maa [5] donne une expression très proche de (5) :

$$
Z_{\mathrm{p}}=\frac{32 \nu \rho_{0} e}{a^{2}} \sqrt{1+\frac{x^{2}}{32}}+j \omega \rho_{0} e\left(1+\frac{1}{\sqrt{3^{2}+\frac{x^{2}}{2}}}\right)
$$




\subsection{Impédance acoustique d'une plaque micro-perforée}

Pour trouver l'expression de l'impédance d'une plaque micro-perforée [3] a fait l'hypothèse que la distance entre les perforations est supposée très petite devant les longueurs d'ondes utilisées. Ceci permet de négliger les réflexions dues à la partie solide de la plaque. D'autre part, il est supposé également que les perforations n'interfèrent pas entre elles, c'est-à-dire que le diamètre de la perforation $a$ est plus petit que l'espacement entre les trous. Ceci permet d'écrire :

$$
Z_{\mathrm{PMP}}=\frac{\Delta P}{\langle u\rangle_{\text {global }}}
$$

Or, avec $S_{\mathrm{p}}$ la surface totale de la plaque et $S_{j \text { perf }}$ la surface de la perforation $j$ :

$$
\langle u\rangle_{\text {global }}=\frac{1}{S_{\mathrm{p}}} \sum_{j=1}^{n \text { perf }} S_{j \text { perf }}\left\langle u_{j}\right\rangle_{\text {perf }}
$$

En supposant que l'onde acoustique traversant la plaque est plane, on peut supposer que les vitesses moyennes d'une perforation à une autre ne varient pas. On a donc :

$$
\langle u\rangle_{\text {global }}=\langle u\rangle_{\text {perf }} \frac{1}{S_{\mathrm{p}}} \sum_{j=1}^{n \text { perf }} S_{j \text { perf }}\langle u\rangle_{\text {perf }} \sigma
$$

L’impédance de la plaque s'écrit alors :

$$
Z_{\mathrm{MPP}}=\frac{\Delta P}{\langle u\rangle_{\text {global }}} \frac{Z_{\mathrm{p}}}{\sigma}
$$

où $\sigma$ désigne le taux de perforation.

\subsection{Les différentes corrections à apporter à l'impédance des PMP}

Les différentes expérimentations réalisées dans ce domaine ont toutes montré la nécessité d'apporter des corrections au modèle théorique. Ces dernières sont liées à la nécessité de tenir compte des effets d'embouchures : les hypothèses sur la forme du champ de vitesse (composante radiale nulle notamment) ne sont évidemment plus acceptables à proximité des embouchures. Ces corrections seront donc d'autant plus importantes que les tubes seront courts (au sens que le champ de vitesse supposé dans le modèle standard n'a pas la possibilité de s'établir).

\section{a) Correction de résistance}

Aux embouchures du tube, les couches limites thermovisqueuses des parois externes de la plaque perforée provoquent une dissipation supplémentaire. Cet effet peut être pris en compte par l'ajout d'un facteur sur la résistance (partie réelle de l'impédance) du tube. Ce terme supplémentaire (ici la correction proposé par

\section{Zone d'interaction}

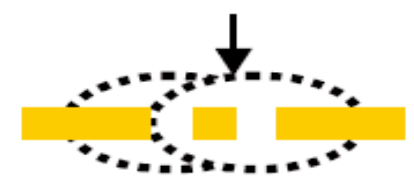

Fig. 1. Deux perforations rapprochées [3].

Ingard $[4,6])$ est mis entre crochet [ ], dans l'expression suivante :

$$
\begin{aligned}
Z_{\text {perf }}= & \frac{32 \nu \rho_{0} e}{a^{2}}\left(\sqrt{1+\frac{x^{2}}{32}}+\left[\frac{\sqrt{2} x a}{8 e}\right]\right) \\
& +j \omega \rho_{0} e\left(1+\frac{1}{\sqrt{3^{2}+\frac{x^{2}}{2}}}\right)
\end{aligned}
$$

\section{b) Correction de réactance}

La masse apparente (partie imaginaire de l'impédance ou réactance) doit également être corrigée.

Par analogie avec le rayonnement d'un piston plan, Rayleigh a établi qu'un ajout de $0,85 R$ à la longueur de tube à chaque embouchure était nécessaire pour le calcul de la réactance. Sivian [7] a remarqué que cette correction de longueur pouvait également être appliquée sur la résistance en lieu et place de la correction de Ingard.

\section{c) Interactions entre perforations}

Il a été observé que ces corrections se traduisant sur la longueur des perforations étaient excessives quand les perforations sont rapprochées (porosité relativement élevée).

Pour prendre en compte ce phénomène, Fok [6] sur la base de considérations géométriques, a proposé une fonction de correction à appliquer sur la correction de longueur de la partie réactive et résistive de l'impédance :

$$
\Delta e_{\mathrm{c}}=\frac{\Delta e}{F_{\mathrm{Fok}}}
$$

$\Delta e$ est la correction d'épaisseur et $F_{\mathrm{Fok}}=\left(1+a_{1} \varepsilon_{\mathrm{F}}+\right.$ $\left.a_{2} \varepsilon_{\mathrm{F}}^{2}+a_{3} \varepsilon_{\mathrm{F}}^{3}+a_{4} \varepsilon_{\mathrm{F}}^{4}+\ldots\right)^{-1}$.

Les premiers coefficients de $F_{\text {Fok }}$ sont :

$$
\left\{\begin{array}{l}
a_{1}=-1,4092 \\
a_{2}=0 \\
a_{3}=0,33818 \\
a_{4}=0 \\
a_{5}=0,06793 \\
a_{6}=-0,02287
\end{array}\right.
$$$$
\varepsilon_{\mathrm{F}}=\frac{a}{\text { entraxe des perforation }} \frac{\sqrt{\pi}}{2}
$$ 
Ainsi, plus le taux de perforation est faible, plus $F_{\text {Fok }}$ se rapprochera de 1 .

L'expression de l'impédance d'une MPP s'écrit alors en tenant compte des trois corrections précédentes :

$$
\begin{aligned}
Z_{\mathrm{p}}= & \frac{32 \nu \rho_{0} e^{\prime}}{a^{2}}\left(\sqrt{1+\frac{x^{2}}{32}}+\frac{\sqrt{2} x a}{8 e^{\prime}}\right) \\
& +j \omega \rho_{0} e^{\prime}\left(1+\frac{1}{\sqrt{3^{2}+\frac{x^{2}}{2}}}\right)
\end{aligned}
$$

avec $e^{\prime}=\left(e+\Delta e_{\mathrm{c}}\right)$.

\section{d) Correction de viscosité}

Dans de nombreux cas, notamment les plaques de petite épaisseur et/ou des trous de petits diamètres, le modèle précédent doit encore être ajusté, les effets dissipatifs étant constatés trop faibles.

Généralement, une correction de viscosité est appliquée. Melling [6] propose une correction d'un facteur 2 mais uniquement dans la longueur interne des trous; Maa [5] applique un facteur 2,33 valable quand les diamètres et épaisseurs sont du même ordre; Dupont [3] corrige directement la résistance d'un facteur variant de 1 à 4 .

Les raisons physiques pouvant justifier cette correction sont à ce jour mal comprises, cette correction est de notre point de vue plus à comprendre et utiliser comme un paramètre de recalage.

\section{Impédance des plaques sandwich micro-perforées}

Dans cette section nous proposons de développer un modèle analytique d'impédance acoustique d'un panneau sandwich composé d'une plaque en nid d'abeille sur laquelle sont collées deux plaques micro-perforées (Fig. 2).

Les matériaux en nid d'abeille ont la particularité d'avoir une structure lacunaire et périodique. Dans un premier temps nous allons résoudre le problème uniquement dans une cellule couplée avec les deux plaques PMP, qui constitue un résonateur de Helmholtz. Après nous généraliserons les résultats à la plaque sandwich toute entière. Pour cela nous allons supposer que les cavités sont de forme cylindrique (on pourra toujours s'y ramener en prenant comme diamètre une dimension caractéristique de celle-ci). On supposera également que le nombre de trous des PMP débouchant dans une cavité est suffisamment grand pour considérer que les impédances des deux portions de plaques en contacts avec celle-ci sont celles des plaques toutes entières.

\section{1 Équations dans la cavité}

Aucun effet thermique et visqueux n'est pris en compte dans la cellule due aux dimensions de celle-ci,

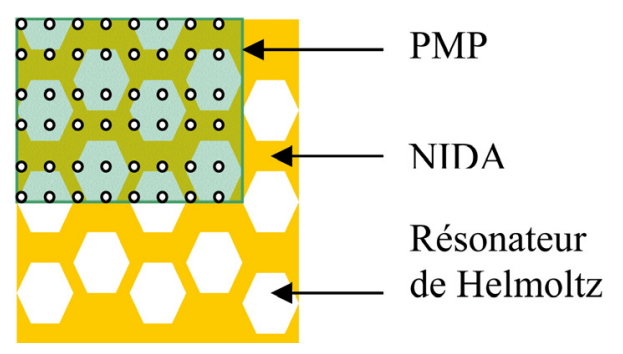

Fig. 2. Représentation du panneau sandwich.

de l'ordre du cm et donc beaucoup plus grandes que les tailles caractéristiques des couches limites thermiques et visqueuses.

En supposant que les excitations sont harmoniques et en utilisant le système de coordonnées cylindriques, les équations linéaires s'écrivent :

$$
\left\{\begin{array}{l}
j \omega \rho_{0} q=-\frac{\partial P}{\partial r} \\
j \omega \rho_{0} u=-\frac{\partial P}{\partial z} \\
\Delta P+\frac{\omega^{2}}{c^{2}} P=0
\end{array}\right.
$$

La troisième équation du système (10) est une équation de Helmholtz dans laquelle $\omega$ et $c$ sont respectivement la pulsation et la célérité du son dans le fluide. Sa résolution nous donne l'expression de la pression acoustique :

$$
\left\{\begin{array}{l}
P=A \mathrm{e}^{-j\left(k_{1} r+k_{2} z\right)}+B \mathrm{e}^{+j\left(k_{1} r+k_{2} z\right)} \\
\sqrt{k_{1}^{2}+k_{2}^{2}}=\frac{\omega}{c}
\end{array}\right.
$$

La première équation de (10) appliquée à la surface de la cavité supposée rigide (vitesse radiale nulle à la surface), montre l'indépendance de la pression vis-à-vis de $r$ et la nullité de la vitesse radiale. On en déduit :

$$
\left\{\begin{array}{l}
\vec{P}=A \mathrm{e}^{-j\left(\frac{\omega}{c} z\right)}+B \mathrm{e}^{j\left(\frac{\omega}{c} z\right)} \\
q=0
\end{array}\right.
$$

La seconde équation de (10) nous permet d'écrire l'expression de la vitesse axiale :

$$
u=\frac{1}{c \rho_{0}}\left(A \mathrm{e}^{-j\left(\frac{\omega}{c} z\right)}-B \mathrm{e}^{j\left(\frac{\omega}{c} z\right)}\right)
$$

\subsection{Propagation acoustique dans la cavité couplée avec deux plaques PMP}

On se place dans le cas où l'onde incidente est normale à la plaque PMP. Les numéros associés aux ondes incidentes, réfléchies et transmises sont utilisés pour repérer les champs de pression et de vitesse dans ces différents cas. 


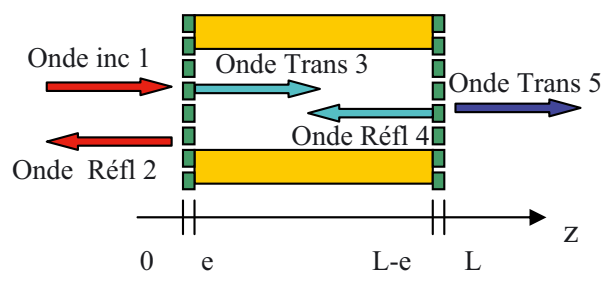

Fig. 3. Paramétrage du système.

Pour résoudre ce problème, nous allons adopter le paramétrage suivant :

Pour l'onde incidente 1 et l'onde réfléchie 2, nous avons :

$$
\left\{\begin{array}{l}
P_{1}=A_{1} \mathrm{e}^{-j\left(\frac{\omega}{c} z\right)} \\
P_{2}=A_{2} \mathrm{e}^{j\left(\frac{\omega}{c} z\right)} \\
u_{1}=\frac{A_{1}}{c \rho_{0}} \mathrm{e}^{-j\left(\frac{\omega}{c} z\right)} \\
u_{2}=-\frac{A_{2}}{c \rho_{0}} \mathrm{e}^{j\left(\frac{\omega}{c} z\right)}
\end{array}\right.
$$

Pour l'onde transmise 3 et l'onde réfléchie 4 , en tenant compte des conditions sur la première plaque PMP, nous avons les deux relations suivantes:

$$
\left\{\begin{array}{l}
A_{3} \mathrm{e}^{-j\left(\frac{\omega}{c} e\right)}-A_{4} \mathrm{e}^{j\left(\frac{\omega}{c} e\right)}=A_{1}-A_{2} \\
A_{3} \mathrm{e}^{-j\left(\frac{\omega}{c} e\right)}+A_{4} \mathrm{e}^{j\left(\frac{\omega}{c} e\right)}=A_{1}+A_{2}+\frac{A_{1}-A_{2}}{c \rho_{0}} Z_{\mathrm{PMP}}
\end{array}\right.
$$

Le même raisonnement avec l'onde transmise 5, nous permet d'aboutir à un système de 4 équations et 5 inconnues.

En les exprimant en fonction de l'amplitude de l'onde de pression incidente 1 , on obtient :

$$
\begin{aligned}
& A_{2}=A_{1} R_{\text {ef }} \\
& A_{3}=A_{1}\left[1+\frac{1-R_{\mathrm{ef}}}{2 c \rho_{0}} Z_{\mathrm{PMP}}\right] \mathrm{e}^{j\left(\frac{\omega}{c} e\right)} \\
& A_{4}=A_{1}\left[R_{\mathrm{ef}}+\frac{1-R_{\mathrm{ef}}}{2 c \rho_{0}} Z_{\mathrm{PMP}}\right] \mathrm{e}^{-j\left(\frac{\omega}{c} e\right)} \\
& A_{5}=A_{1}\left[\begin{array}{l}
\left(1+\frac{1-R_{\mathrm{ef}}}{2 c \rho_{0}} Z_{\mathrm{PMP}}\right) \mathrm{e}^{2 j\left(\frac{\omega}{c} e\right)} \\
-\left(R_{\mathrm{ef}}+\frac{1-R_{\mathrm{ef}}}{2 c \rho_{0}} Z_{\mathrm{PMP}}\right) \mathrm{e}^{2 j \frac{\omega}{c}(L-e)}
\end{array}\right]
\end{aligned}
$$

avec :

$$
\begin{gathered}
R_{\mathrm{ef}}=\frac{Z_{\mathrm{PMP}}}{c \rho_{0}} C \\
C=\left[\frac{\left(\frac{Z_{\mathrm{PMP}}}{c \rho_{0}}-2\right) \mathrm{e}^{2 j \frac{\omega}{c}(L-2 e)}-\left(2+\frac{Z_{\mathrm{PMP}}}{c \rho_{0}}\right)}{\left(\frac{Z_{\mathrm{PMP}}}{c \rho_{0}}-2\right)^{2} \mathrm{e}^{2 j \frac{\omega}{c}(L-2 e)}-\left(\frac{Z_{\mathrm{PMP}}}{c \rho_{0}}\right)^{2}}\right]
\end{gathered}
$$

\subsection{Impédance acoustique d'une cellule couplée avec les PMP}

L'amplitude de la pression à l'interface milieu extérieur/plaque sandwich est l'amplitude de la pression en $z=0$ :

$$
\begin{aligned}
P_{\text {interface }} & =P_{1}(0)+P_{2}(0) \\
& =A_{1}\left(1+R_{\mathrm{ef}}\right)
\end{aligned}
$$

De la même manière l'amplitude de la vitesse vibratoire à l'interface s'écrit :

$$
V_{\text {fluide }}=\frac{A_{1}}{c \rho_{0}}\left(1-R_{\mathrm{ef}}\right)
$$

On définit l'impédance acoustique d'une cellule couplée avec deux PMP comme le rapport entre la pression à l'interface et la vitesse vibratoire :

$$
Z_{\text {cellule }}=c \rho_{0} \frac{1+R_{\mathrm{ef}}}{1-R_{\mathrm{ef}}}
$$

\subsection{Impédance acoustique de la plaque sandwich}

Comme pour le calcul de l'impédance d'une PMP, nous définissons un taux de vide analogue au taux de perforation qui s'exprime de la manière suivante :

$$
\Sigma=\frac{1}{S_{\text {plaque }}} \sum_{j=1}^{n \text { cavité }} S_{j \text { cavité }}
$$

avec $S_{\text {plaque }}$ la surface totale de la plaque et $S_{\text {jcavité la }}$ surface de la cellule numéro $j$. L'impédance acoustique de la plaque sandwich s'écrit alors :

$$
Z_{\text {plaque }}=\frac{Z_{\text {cellule }}}{\Sigma}
$$

\subsection{Impédance d'une plaque sandwich « mince »}

Nous allons supposer dans le cadre de cette étude que les plaques sandwich sont minces. On va donc négliger leur épaisseur et tenter de reformuler l'impédance en utilisant le saut de pression entre les deux faces de celles-ci. Cette hypothèse est justifiée par le fait que l'étude se limite à des ondes acoustiques de faibles fréquences. Les longueurs d'ondes sont alors plus grandes que l'épaisseur des plaques. De plus le modèle mécanique, qui sera utilisé pour les calculs sur une antenne satellite, utilise lui aussi l'hypothèse des coques minces, ce qui justifie davantage cette approche.

Dans ce cas nous pouvons supposer que $\frac{\omega}{c} e$ et $\frac{\omega}{c} L$ sont négligeables devant $\pi$. Les équations (15) deviennent alors dans une cellule de la plaque :

$$
\left\{\begin{array}{l}
A_{2}=A_{1} R_{\mathrm{ef}} \\
A_{3}=A_{1}\left[1+\frac{1-R_{\mathrm{ef}}}{2 c \rho_{0}} Z_{\mathrm{PMP}}\right] \\
A_{4}=A_{1}\left[R_{\mathrm{ef}}+\frac{1-R_{\mathrm{ef}}}{2 c \rho_{0}} Z_{\mathrm{PMP}}\right] \\
A_{5}=A_{1}\left[1-R_{\mathrm{ef}}\right] \\
R_{\mathrm{ef}}=\frac{Z_{\mathrm{PMP}}}{Z_{\mathrm{PMP}}-c \rho_{0}}
\end{array}\right.
$$

Ce qui permet de déduire l'égalité des vitesses normales sur les faces avant et arrière des cavités :

$$
\left\{\begin{array}{l}
V_{\text {fluide-avant }}=\frac{A_{1}-A_{2}}{c \rho_{0}}=A_{1} \frac{\left(1-R_{\mathrm{ef}}\right)}{c \rho_{0}} \\
V_{\text {fluide-arière }}=\frac{A_{5}}{c \rho_{0}}=A_{1} \frac{\left(1-R_{\mathrm{ef}}\right)}{c \rho_{0}}
\end{array}\right.
$$




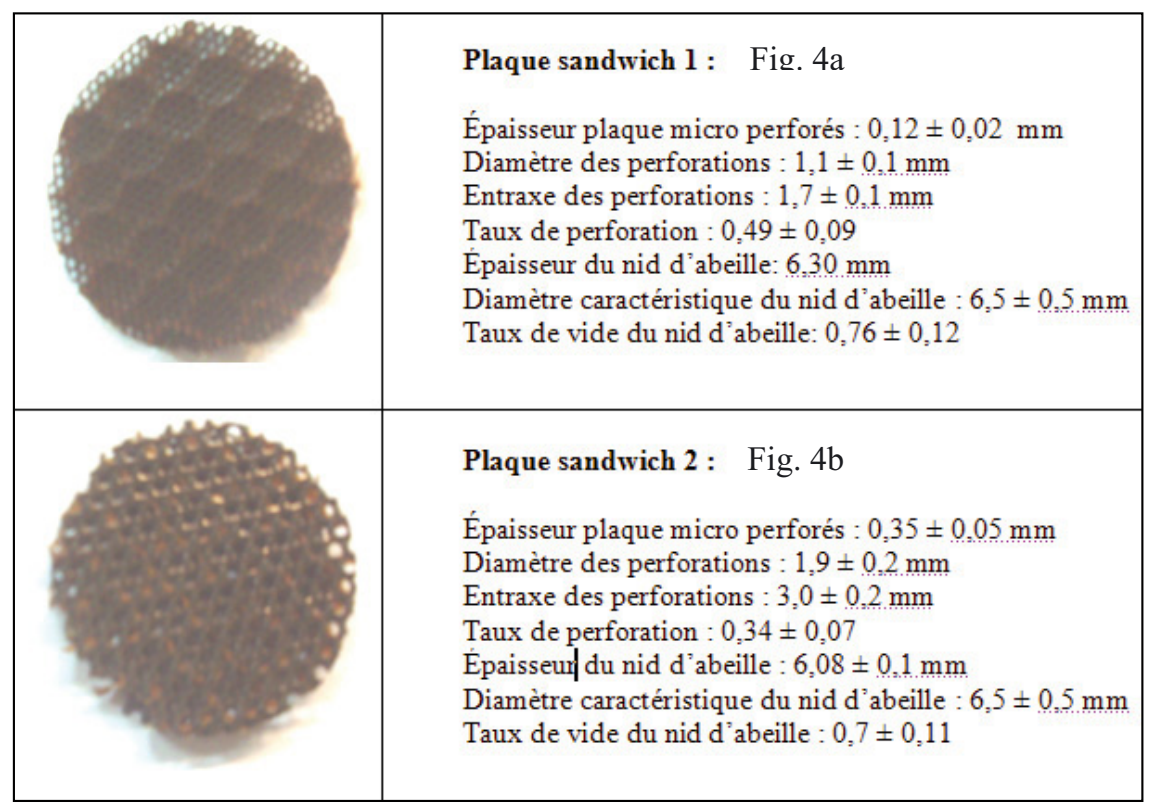

Fig. 4. Caractéristiques géométriques des plaques sandwich.

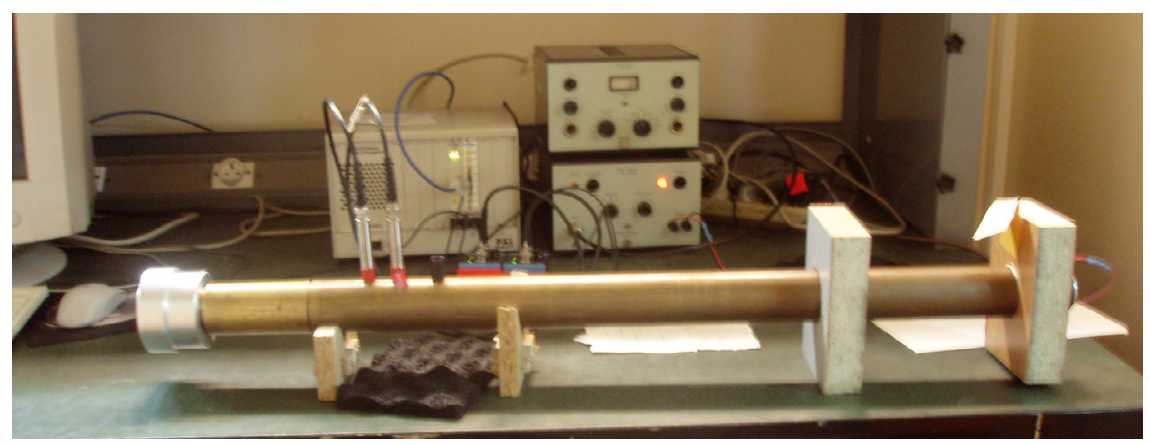

Fig. 5. Dispositif expérimental (tube de Kundt).

Et également l'expression du saut de pression de la cavité :

$$
\begin{aligned}
\Delta P_{\text {cavité }} & =A_{5}-\left(A_{1}+A_{2}\right) \\
& =A_{1}\left(1-R_{\mathrm{ef}}\right)+A_{1}\left(-1-R_{\mathrm{ef}}\right) \\
& =-2 A_{1} R_{\mathrm{ef}}
\end{aligned}
$$

L'impédance s'écrit alors :

$$
\begin{aligned}
Z_{\text {sandwich }} & =\frac{1}{\Sigma} \frac{\Delta P_{\text {cellule }}}{V_{\text {fluide-avant }}} \\
& =\frac{2 R_{\text {ef }}}{\left(R_{\text {ef }}-1\right)} \frac{1}{\Sigma} c \rho_{0}
\end{aligned}
$$

\section{Vérification expérimentale}

Nous disposons de deux échantillons de plaque sandwich micro-perforées, figures $4 \mathrm{a}$ et $4 \mathrm{~b}$ et d'un modèle analytique d'impédance brut défini par l'équation (19). Il reste à valider le modèle analytique expérimentalement à l'aide de mesures de l'absorption en tube de Kundt, figure 5. Le but de cette validation est d'apporter les corrections nécessaires au modèle analytique afin d'obtenir un bon recalage. Nous terminerons par une étude de sensibilité du modèle vis-à-vis des paramètres géométriques de la plaque. Cette dernière est essentielle, car les incertitudes sur certaines grandeurs peuvent conduire à des variations importantes. Nous nous limitons à une fréquence maximale de $2000 \mathrm{~Hz}$. Les dimensions des plaques sandwich étudiés ont été mesurées au pied à coulisse, d'où une certaine incertitude.

\subsection{Mesures au tube de Kundt}

Le coefficient de réflexion $R$ du matériau est déterminé par :

$$
R=\left(\frac{H-\mathrm{e}^{-j k s}}{\mathrm{e}^{j k s}-H}\right) \mathrm{e}^{2 k(1+s)}
$$

avec :

$H$ : fonction de transfert des 2 microphones;

$k$ : nombre d'onde;

$s$ : espacement entre les micros;

$l$ : distance entre la surface du matériau et le micro le plus proche de l'échantillon. 


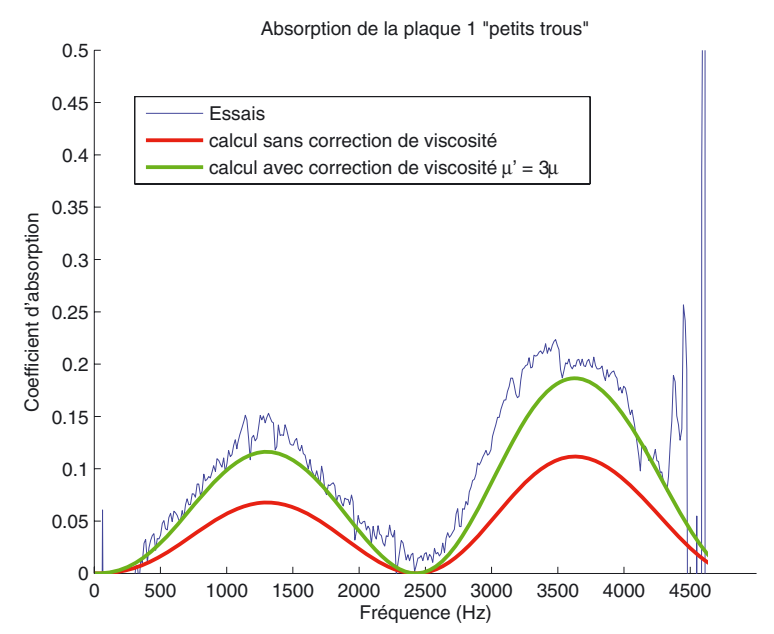

Fig. 6. Absorption correspondante au sandwich 1 (petits trous).

Le coefficient de réflexion permet ensuite d'accéder au coefficient d'absorption $\alpha$ et à l'impédance de surface caractéristique par les relations

$$
\alpha=1-\left|R^{2}\right| \text { et } \frac{Z}{\rho c}=\frac{1+R}{1-R}
$$

Pour effectuer les mesures en absorption des plaques sandwich, figures 6 et 7 , nous avons laissé entre la paroi rigide et l'échantillon une cavité d'air dont l'impédance est donnée par :

$$
Z_{\text {cavié }}=-j \omega c \operatorname{cotan}\left(\frac{\omega l}{c}\right)
$$

$l$ : représente la profondeur de la cavité de $6 \mathrm{~cm}$.

\subsection{Résultats expérimentaux et comparaison avec le modèle}

Le système de mesure, figure 5, livre l'impédance de surface et aussi le coefficient d'absorption $\alpha$ du système formé par l'échantillon et la cavité.

Pour faire la comparaison entre les résultats analytiques et ceux des essais, les corrections de résistance, de la réactance et d'interférence entre les trous sont appliquées. La correction de la viscosité est utilisée pour recaler les courbes d'absorption.

Les figures 6 et 7 montrent la comparaison entre les absorptions analytiques (avec et sans correction de la viscosité) et l'absorption mesurée. Dans le cas du sandwich avec les gros trous (Fig. 7), l'utilisation d'un facteur de correction de viscosité égale à 3 surestime l'absorption calculée. Dans ce cas, un facteur de correction plus faible égal à 1,8 est suffisant. Cette constatation est cohérente avec la physique, car il y a moins de dissipation par frottement lié à la viscosité pour des trous de plus grand diamètre $(2 \mathrm{~mm})$ que pour des trous de petit diamètre (1 mm).

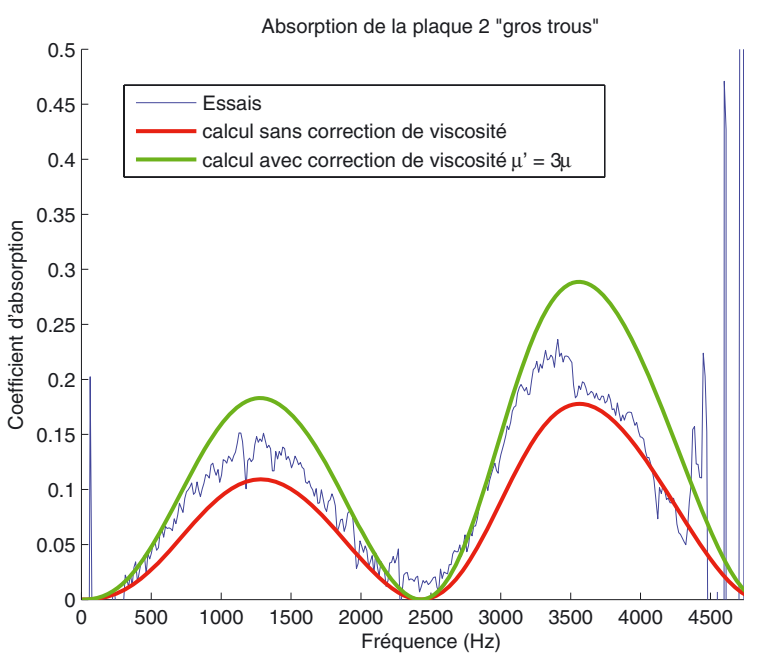

Fig. 7. Absorption correspondante au sandwich 2 (gros trous).

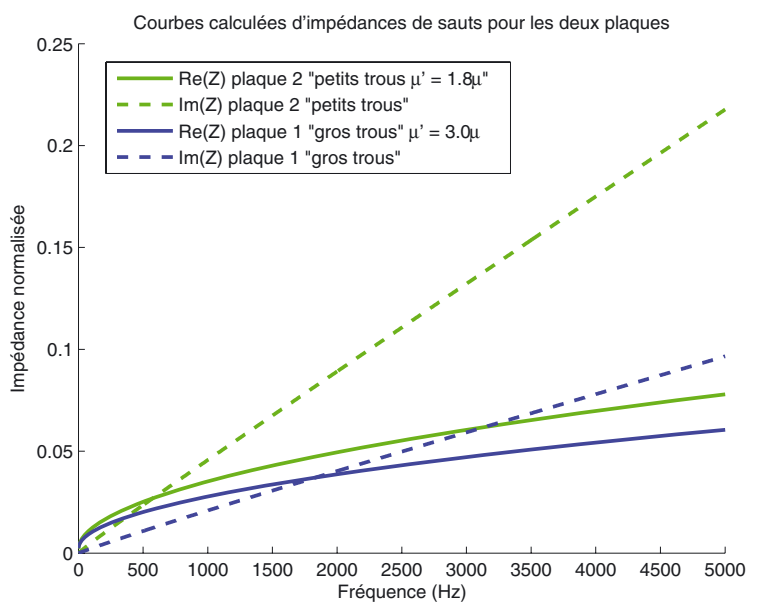

Fig. 8. Courbes d'impédances de saut correspondant aux deux plaques sandwich en utilisant des corrections de viscosité recalées.

Les résultats obtenus montrent que le modèle analytique développé est valable jusqu'à la fréquence de $2500 \mathrm{~Hz}$, ce qui est largement suffisant pour être utilisé dans des calculs prédictifs en basses fréquences $[0-2 \mathrm{kHz}]$.

\section{3 Étude de la sensibilité du modèle développé}

Les paramètres géométriques ayant servi pour la comparaison sont entachés d'erreur (Fig. 4). Nous allons donc étudier l'influence de leurs variations en observant leurs effets sur l'absorption. Nous définissons une erreur relative :

$$
\operatorname{ERREUR}=\frac{U_{\max }-U_{\min }}{U_{\text {moyeni }}}
$$

où $U_{\max }$ et $U_{\min }$ désignent respectivement la valeur maxi et la valeur mini de l'absorption obtenue en faisant varier les paramètres. $U_{\text {moy }}$ est la valeur de l'absorption obtenue avec les paramètres nominaux. 


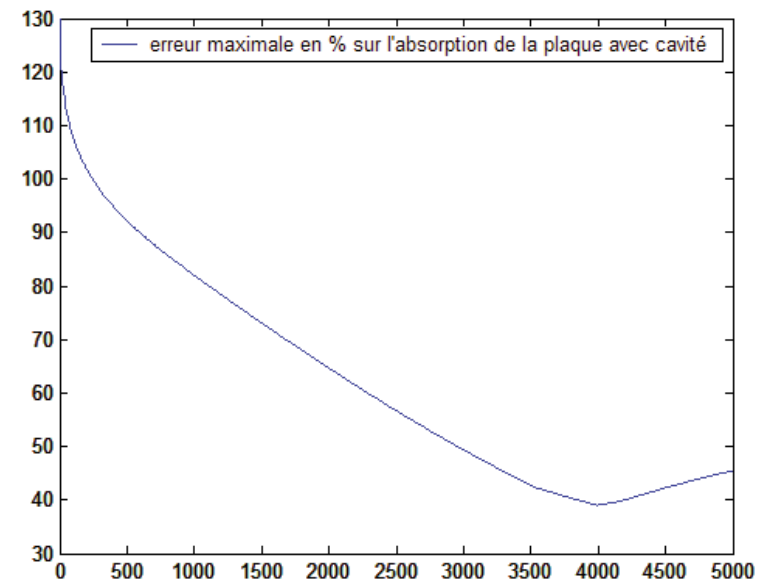

Fig. 9. Erreur maximale commise sur le calcul de l'absorption.

Cette courbe montre une dispersion importante de l'absorption calculée. Elle correspond à l'erreur maximale produite par les incertitudes maximales sur les paramètres géométriques. Il est à noter que les incertitudes les plus influentes sur l'erreur de l'absorption sont celles liées au diamètre des trous et à la densité des perforations.

\section{Conclusion et perspectives}

Nous avons développé un modèle analytique d'impédance acoustique de saut et d'absorption homogénéisée, adapté aux plaques sandwich micro-perforées formées par un cœur en NIDA recouvert par deux plaques micro-perforées (PMP). Ce modèle est basé sur celui établi pour les plaques simples micro-perforées disponibles dans la littérature.
Le modèle ainsi développé et validé par les essais, sera utilisé pour le calcul de la réponse vibro-acoustique de structures spatiales (antenne de satellite) à base de PMP en matériaux composites sandwich à cœur en NIDA, à l'aide de la méthode des éléments finis et éléments finis de frontières implémentées dans le code RAYON (I-DEAS Vibro-Acoustics).

\section{Références}

[1] F. Asdrubali, G. Pispola, Properties of transparent sound-absorbing panels for use in noise barriers, JASA 121 (2007) 214-221

[2] L.B. Crandall, Theory of vibrating systems and sound, New York, Van Nostran, 1926, 229 p.

[3] T. Dupont, Transparence et absorption acoustiques des structures micro perforées, Ph.D. Thesis, Institut National des Sciences Appliquées, Lyon, 2002

[4] U. Ingard, On the theory and design of acoustic resonators, JASA 25 (1953) 1037-1061

[5] D.-Y. Maa, Potential of micro perforated panel absorber, JASA 104 (1998) 2861-2866

[6] T.H. Melling, The acoustic impedance of perforates at medium and high sound pressure levels, J. Sound Vib. 29 (1973) 1-65

[7] L.J. Sivian, Acoustic Impedance of small orifices, JASA 7 (1935) 94-101

[8] M.R. Stinson, Acoustic impedance of small circular orifice in thin plates, JASA 77 (1985) 2039-2042

[9] M.R. Stinson, The propagation of plane sound waves in narrow and wide circular tubes, and generalization to uniform tubes of arbitrary cross-sectional shape, JASA 89 (1991) 550-558

[10] G.B. Thurston, Periodic flow through circular orifices, JASA 25 (1953) 26-31 